\title{
NOVOS PRODUTOS PARA O TRATAMENTO PRESERVANTE DA MADEIRA. "PERSPECTIVAS DA PESQUISA E UTILIZAÇÃO"
}

\author{
Martha Andreia Brand*, José Anzaldo**, João Carlos Moreschi*** \\ *Eng ${ }^{\text {a }}$. Florestal, M.Sc., Doutoranda em Eng. Florestal, UFPR, UNIPLAC - martha@uniplac.net \\ **Eng. Químico, M.Sc., Doutorando em Eng. Florestal, UFPR - janzaldo@hotmail.com \\ ***Eng. Florestal, Dr., Depto. de Engenharia e Tecnologia Florestal, UFPR - moreschi@membracel.com.br
}

Recebido para publicação: 28/07/2005 - Aceito para publicação: 03/03/2006

\begin{abstract}
Resumo
O aumento da preocupação com as questões ambientais e de saúde dos operadores de processos de tratamento preservante e dos consumidores da madeira tratada tem gerado a necessidade de desenvolvimento de novos produtos. Nesse sentido, foi realizada uma revisão bibliográfica para verificar qual a tendência das pesquisas e o que vem acontecendo com os produtos já existentes no mercado. Assim, este trabalho apresenta informações sobre produtos voltados principalmente para substâncias extraídas das próprias plantas, uso de microorganismos como agentes de proteção, substâncias inibidoras da ação enzimática dos agentes xilófagos e produtos que utilizem princípios de tratamento menos impactantes ambientalmente. Pode-se concluir que o direcionamento das pesquisas tem objetivado sempre a redução dos impactos ambientais e os cuidados com a saúde das pessoas e outros animais. Além disso, um ponto que precisa ser desenvolvido são as metodologias de teste de novos produtos, para a redução do tempo e dos custos para o seu registro, sem que seja comprometida a qualidade dos novos produtos preservantes da madeira.

Palavras-chave: Biocidas; eficiência; composição.
\end{abstract}

\begin{abstract}
Present situation of research and utilization of new products for wood preserving treatment. The increasing concearning on issues related to environment protection and wood treatment workers and preserved wood users brought the necessity of developing new products. Thus, a bibliographic research was done in order to verify research tendencies and to increase the knowledge about products already on the market. Based on this facts, this work presents information about products, specially of substances extracted from plants; the use of microorganisms as protection agents; inhibiting substances of the enzymatic action of organisms that attack wood; and other environmentally less aggressive products. It can be concluded that the aiming of the research has objectified always the reduction of the ambient impacts and the cares with the health of the people and other animals. Moreover, a point that it needs to be developed is the methodologies of test of the new products for the reduction of the time and the costs for the register of the same ones, without that the quality of the new products for the wood is compromised.

Keywords: Biocides; efficiency; chemical composition.
\end{abstract}

\section{INTRODUÇ̃̃O}

A demanda do mundo por biocidas crescerá 5,6\% anualmente a partir de 2006 (FREEDONIA GROUP, 2004). Aliado ao crescimento da demanda, as regiões mais desenvolvidas do mundo estão sendo regidas por legislações cada vez mais exigentes com relação aos produtos biocidas, restringindo ou proibindo o uso de alguns produtos tradicionalmente utilizados.

Nessas regiões, a indústria dos biocidas é afetada substancialmente por órgãos reguladores. Nos Estados Unidos, a Agência de Proteção Ambiental requer que os biocidas sejam sujeitos ao registro do produto como pesticida, que é um procedimento caro e demorado. A diretriz orientadora dos produtos biocidas da União Européia entrou em vigor no ano 2000 e removerá um grande número de produtos do mercado se estes não passarem pelo procedimento de regulamentação. Em diversos países, os regulamentos a respeito do uso dos biocidas estão começando a assemelhar-se àqueles dos Estados 
Unidos, Canadá, Japão e Europa Ocidental. Em áreas menos desenvolvidas do mundo, os regulamentos são geralmente menos restritivos (WORLD BIOCIDES, 2004).

Assim, o desenvolvimento de métodos que permitam uma avaliação mais rápida dos novos produtos químicos, para substituição dos testes de campo com grande período de tempo, e a obtenção de produtos que tenham baixa toxicidade para mamíferos, com baixo impacto ambiental, são alguns dos fatores fundamentais que devem ser considerados para o sucesso técnico, econômico e ambiental de novos produtos preservantes da madeira (STRATEGIS, 2004).

De forma geral, o desenvolvimento de pesquisas sobre preservantes de madeira está em uma fase crucial, sendo necessário o seu direcionamento para a análise de produtos com menor potencial de danos ambientais e à saúde de outros organismos. Porém, apesar da eficiência comprovada de algumas alternativas ambientalmente corretas para o tratamento da madeira, as informações sobre sua viabilidade econômica ainda são difíceis de serem encontradas, ou ainda nem começaram a ser avaliadas.

Assim, este trabalho apresenta informações de pesquisas recentes de produtos preservantes da madeira, voltadas principalmente para substâncias extraídas das próprias plantas, ao uso de microorganismos como agentes de proteção, para substâncias inibidoras da ação enzimática dos agentes xilófagos e para produtos que utilizem princípios de tratamento menos impactantes ambientalmente.

\section{Situação atual dos produtos preservantes tradicionais}

Com relação aos produtos hoje existentes no mercado, o arseniato de cobre cromatado (CCA), patenteado em 1934, é um preservativo altamente eficaz. Protege a madeira da podridão por fungos, cupins, agentes marinhos e da luz ultravioleta. Repelentes de água podem ser adicionados ao CCA para melhorar sua resistência à absorção de umidade pela madeira. No entanto, alguns críticos consideram que o CCA pode causar efeitos adversos ao ambiente e danos à saúde dos homens (STRATEGIS, 2004), embora o Conselho Americano da Ciência e da Saúde e a Agência de Proteção Ambiental, ambos dos Estados Unidos, tenham concluído que a madeira tratada com CCA não apresenta nenhum perigo para a saúde. Entretanto, as preocupações recentes com relação à lixiviação do arsênio e as dúvidas sobre a eliminação dos resíduos da madeira tratada com CCA afetarão cada vez mais as atitudes dos consumidores em relação ao uso da madeira tratada com este produto (FOREST AND WILDLIFE, 2004a).

Ainda assim, não se pode deixar de levar em consideração que o CCA é usado para tratar aproximadamente $80 \%$ de todos os produtos de madeira nos Estados Unidos e é essencialmente o único preservante usado na construção civil. Conseqüentemente, existe grande necessidade de desenvolvimento de produtos para tratar a madeira ambientalmente mais seguros e que tenham preços competitivos com o CCA (FOREST AND WILDLIFE, 2004a).

Vários preservantes livres de cromo e arsênio foram introduzidos em várias partes do mundo, na tentativa de substituir o CCA. Esses contêm tipicamente a etanolamina de cobre, um co-biocida orgânico com baixa toxicidade para mamíferos. Os exemplos são o quat de cobre amoniacal (ACQ) e o cobre azole (CBA), que foram usados por alguns anos pela Europa e Japão, sendo que o ACQ foi usado também nos Estados Unidos. Porém, são consideravelmente mais caros do que o CCA (STRATEGIS, 2004).

Dentro desse contexto, se necessário, a indústria adotará sistemas de co-biocidas orgânicos de cobre amina ou então coquetéis orgânicos, mas a taxa de mudança será determinada pela demanda dos consumidores e pela resposta resultante dos órgãos reguladores (STRATEGIS, 2004). Entretanto, a presença do cobre limitará provavelmente o uso a longo prazo desses preservantes (FOREST AND WILDLIFE, 2004a).

Alguns produtos baseados em biocidas orgânicos estão sendo desenvolvidos, mas o custo deles é muito maior do que o do CCA (FOREST AND WILDLIFE, 2004a), o que leva ao uso atual de pequenas quantidades. Além disso, sua vantagem principal é também sua desvantagem, pois são biodegradáveis não somente no solo, mas também na madeira (STRATEGIS, 2004).

$\mathrm{O}$ arseniato de cobre amoniacal do zinco (ACZA) vem sendo introduzido no Canadá como um substituto para o arseniato de cobre amoniacal (ACA). Somente uma planta de tratamento canadense está usando esse preservante, primeiramente para aplicações marinhas e madeiras de grandes dimensões (STRATEGIS, 2004).

Com relação ao pentaclorofenol, esse produto ainda é usado em alguns serviços públicos no Canadá Central, e o creosoto é usado para dormentes de estradas de ferro (STRATEGIS, 2004), como também é comum o uso desses produtos em outros países do mundo. 
Os preservativos baseados em borato de sódio não são atualmente capazes de substituir os produtos à base de cobre ou os óleossolúveis, porque não se fixam bem à madeira. Os boratos recentemente foram introduzidos no Canadá, sendo utilizados principalmente para mercados de exportação, em que a resistência a cupins é importante. Os boratos têm inúmeras vantagens relacionadas à proteção da madeira contra as podridões, cupins, besouros e fogo (em concentrações mais elevadas). São também incolores, inodoros, baratos e têm baixa toxicidade para mamíferos. Eles têm ainda alta difusão, podendo ser aplicados em madeira serrada verde, com penetração em toda a profundidade da peça. A desvantagem principal desses produtos é a baixa retenção; conseqüentemente, a madeira tratada não deve ser utilizada em ambientes úmidos. A fixação dos boratos foi tentada por vários laboratórios em todo o mundo, mas nenhuma solução prática foi identificada ainda (STRATEGIS, 2004).

Strategis (2004) considera que os produtos boratados têm grande potencial, mas técnicas precisam ser desenvolvidas, com custos satisfatórios, para fixar esses produtos na madeira, sem afetar seu potencial de preservação. Para os biocidas orgânicos, devem ser desenvolvidas combinações com custos aceitáveis, que tornem o produto estável na madeira, biodegradável no solo e que queimem sem gerar emissões ou cinza tóxica.

\section{Produtos preservantes baseados em substâncias biocidas produzidas pelas plantas}

Os danos ambientais e à saúde das pessoas que manipulam os produtos preservantes tradicionais têm preocupado o mundo e feito com que seja despertado o interesse por pesquisas que desenvolvam produtos naturais para o tratamento da madeira (ONUORAH, 2000).

Dentro dessa perspectiva, e como parte do programa para desenvolver novos preservantes de madeira, Forest and Wildlife (2004a) estudou o motivo pelo qual determinadas madeiras são naturalmente duráveis. Nesse sentido, Schultz e Nicholas (2002) também concordam que uma forma de desenvolvimento de novos preservantes de madeira é estudar o cerne das espécies que apresentam durabilidade natural alta, desde o entendimento das causas da durabilidade natural até a sugestão de formas alternativas de proteger a madeira. Assim, esses autores têm estudado o papel que os extrativos, particularmente os estilbenóides, têm na durabilidade natural do cerne.

Schultz e Nicholas (2002) sugerem que os extrativos podem proteger o cerne do ataque de fungos, e que outras substâncias não-biocidas, como os antioxidantes, trabalham juntas com os extrativos para proteger o cerne da degradação. Forest and Wildlife (2004a) acrescenta que esses extrativos possuem também agentes quelantes, além de repelentes de água. Os quelantes referem-se à habilidade dos extrativos fenólicos em formar complexos com metais (HILLIS; SUMINOTO, 1989'; ${ }^{1}$ SLABERT, 1992²; COOPER-DRIVER; BHATTCHARYA, 1998³, citados por SCHULTZ; NICHOLAS, 2002).

Schultz e Nicholas (2002) citam ainda Green et al. (1997) e Erikson et al. (1990) quando mencionam que a degradação da madeira por fungos freqüentemente envolve vários metais, ou na forma livre ou como componentes chave de enzimas. Conseqüentemente, o quelante pode ser uma forma adicional pela qual os extrativos protegem a madeira, e poderiam agir sinergicamente, combinados com as propriedades antioxidantes e fungicidas estudadas anteriormente.

No estudo feito por Schultz e Nicholas (2002), os pesquisadores testaram o trinômio fungicida (Propiconazole), antioxidante (BHT e ácido tânico) e quelante (3\% de sal trisódico do N,N" - $1,2-$ etanedilbis [N-carboxil-metil) glicine] (tri-Na EDTA) e 1,10 phenantroline), em madeira de pinus e álamo, com o objetivo de testar seu potencial preservante em duas espécies de madeira expostas a fungos de podridão parda (Gloeophyllum trabeum) e branca (Trametes versicolor).

Os resultados desses testes demonstraram que o biocida sozinho tem pouca eficiência no controle das podridões. Porém, as maiores concentrações do biocida (concentração de $0,12 \%$ ) $+5 \%$ de BHT e + $3 \%$ de ácido tânico apresentaram melhor controle sobre as podridões. Além disso, o uso somente do biocida e do ácido tânico foi o segundo melhor resultado obtido. Mesmo em outros testes feitos pelos mesmos autores, substituindo-se o biocida, os antioxidantes e o metal-complexante, sempre os melhores resultados foram obtidos nas combinações das maiores concentrações do biocida, quando este foi aliado aos outros componentes de teste.

\footnotetext{
${ }^{1}$ Hillis, W. E., Suminoto, M. Effect of extractives on pulping. In: Rowe, J.W. (Ed.) Natural products of wood plants II. New York: Springer-Verlag, 1989.p. 880-920.

${ }^{2}$ Slabert, N. Completxation of condensed tannins with metal íons. In: Hemingway, R. W., Laks, P.E. (Eds.) Plant polyphenols. New York: Plenum Press, 1992. p. 421-436.

${ }^{3}$ Cooper-Driver, G.A., Bhattcharya, M. Role of phenolics in plant evolution. Phytochemistry 49. 1998.
} 
De maneira geral, encontra-se em Forest and Wildlife (2004a) que, quando um quelante ou um antioxidante é adicionado sozinho à madeira, não ocorre nenhum efeito protetor. Entretanto, quando um antioxidante e um quelante, ou ambos, são combinados com uma variedade de biocidas orgânicos comercialmente utilizados, há um aumento na eficiência (isto é, um efeito sinérgico), quando comparado com o biocida orgânico sozinho, no mesmo nível. $\mathrm{O}$ aumento na eficiência é observado tipicamente quando os três componentes (biocida orgânico, antioxidante e quelante) estão atuando juntos.

Muitos desses aditivos não-biocidas têm custo baixo e são ambientalmente menos agressivos, como por exemplo o BHT ou o galatepropil, que são aditivos de alimento aprovados. Assim, como o efeito sinérgico entre biocidas orgânicos, antioxidantes e quelantes já foi comprovado cientificamente, o custo do tratamento da madeira pode ser reduzido, além de serem minimizados os efeitos ambientais adversos do uso isolado do biocida orgânico (FOREST AND WILDLIFE, 2004a).

Onuorah (2000) também trabalhou com substâncias naturais extraídas das plantas para o uso como preservante, testando a eficiência dos extrativos do cerne de duas espécies de árvores nigerianas (Milicia excelsa e Erythrophleum suaveolens), quando aplicados em blocos de madeira de uma espécie não durável, contra fungos de podridão parda e branca.

Nesse estudo, concluiu-se que níveis de retenção entre 48,056 a $96,111 \mathrm{~kg} / \mathrm{m}^{3}$ dos extrativos testados conferiram à madeira tratada resistência aos fungos de podridão branca e parda. Porém, níveis de retenção baixos $\left(8,009\right.$ a $\left.24,778 \mathrm{~kg} / \mathrm{cm}^{3}\right)$ dos extrativos não foram efetivos. Além disso, a podridão branca apresentou maior tolerância à ação dos extrativos (ONUORAH, 2000).

Outro trabalho interessante resulta da aplicação que fizeram Celimene et al. (1999). Os autores analisaram o efeito de três compostos do tipo estilbenóide, derivados da pinosilvina, os quais foram utilizados como preservantes em madeiras de folhosas e coníferas. As madeiras foram tratadas com uma solução formada por partes iguais de pinosilvina, monometileter-pinosilvina e dimetileter-pinosilvina. Os resultados obtidos indicaram que essa mistura de compostos apresentou efeito fungicida contra os fungos de podridão parda, mas não foi efetiva para o ataque dos fungos de podridão branca.

Outro exemplo é o trabalho de Kartal et al. (2004), que usaram os compostos fenólicos resultantes da obtenção de um biocombustível. Esse agente protetor foi testado em madeira de Pinus sp., demonstrando ser efetivo na prevenção de ataque de fungos de podridão parda. Porém, infelizmente o teor de vanilina, ácidos láctico e acético promoveram o ataque de cupins. Apesar disso, os autores concluíram que com uma boa separação desses compostos, este ou outros produtos similares podem ter futuro como preservantes de madeiras.

Na procura de agentes com um menor impacto ao ambiente, Voda et al. (2003) usaram alguns óleos essenciais como preservantes de madeiras. Os autores observaram que compostos fenólicos, feniléteres e aldeídos aromáticos atuam como inibidores do desenvolvimento de fungos manchadores e de podridão parda. Os compostos testados foram uma mistura de eugenol, vanilina, cedreno e cafeno, entre outros. Porém, os estudos não comprovaram eficiência contra os cupins.

Outra linha de pesquisa está sendo a análise da resina extraída da espécie Parthenium argentatum Gray. Esse extrativo foi testado por Bultman et al. (1991) para o controle de agentes marinhos (brocas e crustáceos), cupins e fungos de podridão mole, branca e parda, degradadores da madeira. Os resultados obtidos indicaram que a madeira foi levemente atacada por limnorias, sendo que se apresentou com coloração mais clara, caracterizando lixiviação da resina. Em contrapartida, as testemunhas não tratadas com a resina apresentaram ataque severo. A madeira não foi atacada por teredinídeos, mas muito atacada por Martesia striata. Com relação aos cupins, para Coptotermes sp. e para Heterotermes sp., constatou-se que a madeira não foi atacada. De maneira geral, a madeira não foi protegida contra o ataque de agentes do gênero Pholas, e a proteção ocorrida contra as limnorias deveu-se em parte às incrustações ocorridas durante o período, impedindo o acesso desses agentes à madeira. A maior eficiência da resina foi, sem dúvida, contra os cupins. Com relação aos fungos, os autores concluíram que existe diferença significativa na diminuição do ataque destes na madeira tratada, em relação à não tratada.

Nakayama et al. (2001) também trabalharam com a resina obtida de Parthenium argentatum como agente preservante da madeira. Porém, esses autores utilizaram, além da resina extraída da planta, a própria planta na produção de painéis reconstituídos, para a realização dos testes de eficiência. O motivo para a verificação de uso da planta, ao invés da aplicação da resina extraída em outras espécies, é o alto custo da extração, que atualmente inviabiliza o processo.

Assim, o teste foi conduzido com madeira impregnada com a resina extraída de Parthenium argentatum e painéis feitos com Parthenium argentatum (com a planta inteira e com resíduos da planta). 
Foram utilizados diferentes processos de colagem nos painéis. Estes foram colados com fenolformoldeído e polietileno de alta densidade ou somente com a resina extraída da planta. As partículas utilizadas para a produção dos painéis foram de dois tipos: partículas da planta que sofreram extração da borracha e tiveram, ao final da extração, $3 \%$ de borracha e $10 \%$ de material resinoso, e partículas sem extração prévia, contendo $6 \%$ de borracha e $10 \%$ de resina da planta (NAKAYAMA et al., 2001).

Os tratamentos foram utilizados em testes laboratoriais acelerados e expostos a cupins e fungos de podridão parda. Os resultados obtidos indicaram que, para a madeira tratada com a resina, é necessária uma impregnação de $50 \%$ ou mais do peso da madeira em resina para que haja controle efetivo de cupins. Além disso, a fabricação de painéis diretamente com a planta é muito mais simples do que extrair a resina e impregnar em outro tipo de madeira. As chapas de partículas da planta sem adesivo (fenol-formoldeído e polietileno de alta densidade) tiveram atividade mediana de cupins, e painéis feitos com galhos tiveram alta mortalidade dos cupins. Todos os tipos de painéis apresentaram resistência a fungos e cupins, mas as chapas feitas sem adesivo (somente contendo a resina da planta) tiveram maior inchamento, indicando a necessidade de adesivos ou repelentes à água (NAKAYAMA et al., 2001).

Pitcher (1992), realizando uma análise econômica do uso da resina de Parthenium argentatum, concluiu que o uso desse componente para a preservação da madeira pode depender do desenvolvimento dos subprodutos desse arbusto. Tecnicamente, a resina se mostrou promissora com preservante e retardante do fogo, porém, ainda é desconhecida a fração da resina que efetivamente é tóxica para os fungos. Esse autor menciona ainda que o sucesso desse método dependerá em parte do estabelecimento de padrões e rendimentos suficientes do arbusto para fornecer a resina. Os custos de produção ainda são desconhecidos, porém os custos de operação seriam mínimos.

\section{Utilização de microorganismos como agentes de proteção da biodegradação}

Croan (1997) avaliou o uso de uma cultura mutante da bactéria Streptomyces rimosus (SC - 36) para o controle da biodegradação da madeira por fungos de podridão parda, mole e branca. O autor testou o microorganismo em laboratório (placas de Petri) e em blocos de madeira de Pinus sp. e Liquidambar styraciflua, como também em testes de campo. Os resultados obtidos nesse trabalho demonstraram que o uso de Streptomyces rimosus foi efetivo no controle da biodegradação da madeira por fungos de podridão parda, branca, mole e ainda por fungos descoloradores, manchadores e emboloradores, tanto em testes de laboratório como em campo.

Segundo Cook e Baker (1983) ${ }^{4}$, citados por Highley (1995), um dos gêneros de fungos mais largamente estudados como agente de biocontrole da degradação da madeira é o Trichoderma, que tem controlado muitos patógenos em condições de teste experimental. Porém, segundo Highley, Padmanabha e Howell (1996), o sucesso do desenvolvimento do uso de agentes biocontroladores para proteger a madeira de fungos requer ainda um conhecimento detalhado do mecanismo empregado pelo agente no controle da biodegradação.

As espécies de Trichoderma produzem um número de agentes antifúngicos bem estabelecidos, como a gliovirina e a gliotoxina. A gliotoxina produzida pelo Trichoderma virens está associada com o biocontrole de algumas doenças de plantas, mas sua importância para o biocontrole dos fungos degradadores da madeira é pouco conhecida (HIGHLEY; PADMANABHA; HOWELL, 1996). Nesse sentido, Highley, Padmanabha e Howell (1996) estudaram a habilidade da gliotoxina (GLT+) isolada, produzida pelo $T$. virens, e da gliotoxina deficiente (GLT-), produzida pela forma mutante do T. virens, para inibir o crescimento de fungos degradadores da madeira. A análise foi feita em ágar, como meio de cultura. Os autores constataram que fungos de podridão parda e branca foram totalmente inibidos pelo GLT+ isolado e parcialmente pelo GLT- mutante. Porém, o GLT+ isolado e o GLT- mutante foram ineficientes na prevenção do crescimento de fungos emboloradores e manchadores. Dessa forma, os resultados obtidos pelos autores indicaram que a gliotoxina não é o mecanismo primário no controle dos fungos degradadores da madeira, mas contribui para o controle, juntamente com outros mecanismos.

Highley (1995) também realizou um estudo no qual testou o Trichoderma virens (GL-21) no controle de fungos de podridão parda e branca, e fungos emboloradores e manchadores foram incluídos no experimento para determinar os efeitos dos metabólitos do GL-21 na inibição do seu crescimento. Os testes foram conduzidos em placas de Petri e em blocos de Pinus e Acer. Os resultados dos testes, em ambos os experimentos, demonstraram que o GL-21 foi efetivo no controle do crescimento dos fungos de podridão parda, branca, manchadores e emboloradores. A esterilização de blocos pré-tratados fez com

\footnotetext{
${ }^{4}$ Cook, R.J., Baker, K. F. The nature and practice of biological control of plant pathogens. St. Paul: Amer. Phytopathol Soc. 1983.
} 
que a biodegradação ocorresse, devido à morte do biocontrolador e à perda dos metabólitos que inibem o crescimento dos fungos biodegradadores da madeira. Esse biocontrolador se mostrou efetivo na inibição do crescimento dos fungos degradadores da madeira e no controle da perda de peso pela degradação.

\section{Produtos inibidores da atividade enzimática dos fungos}

A presença de ácido oxálico tem sido observada durante o ataque de fungos de podridão parda, por vários autores como Hyde e Wood $(1995)^{5}$, Backa et al. (1992) ${ }^{6}$, Espejo e Agosin (1991) ${ }^{7}$, Green et al. $(1991)^{8}$, Schmidt et al. $(1981)^{9}$, citados por Green III et al. (1997). A formação de ácido oxálico provoca a hidrólise das polioses da parede celular da madeira, devido à geração de um $\mathrm{pH}$ ácido. A hidrólise forma orifícios por onde a entrada das hifas dos fungos é facilitada.

O oxalato é reportado por estar integralmente envolvido, direta ou indiretamente, na formação dos radicais hidróxi ou peróxi. O oxalato pode agir como um agente redutor para a conversão do $\mathrm{Fe}^{3}$ em $\mathrm{Fe}^{2}$, requerido para a Fenton química que despolimeriza os polissacarídeos (SCHMIDT et al., 1981; BACKA et al., 1992; SUTTIE et al., 1996).

Green III et al. (1997), com base na análise de trabalhos de autores como Green et al. (1995 a,b), Evans et al. (1994), Beth-Andersen et al. (1993), Wang et al. (1992) e Beth-Andersen (1987), mencionam que o ácido oxálico está também envolvido na formação de complexos de outros cátions, como $\mathrm{Ca}^{2+}$, especialmente do pectato de cálcio na membrana das pontuações, componentes da lamela média e células de raio, como tmabém na complexação do zinco.

Assim, tem sido investigada a utilização de agentes precipitantes do $\mathrm{Ca}^{2+}$ para inibir a degradação da madeira por fungos. Um desses produtos é o N,N-naftaloilhidroxi lamina (NHA), primeiramente usado por Beck $(1951)^{10}$, citado por Green III et al. (1997), para a determinação do soro cálcico, que tem se mostrado um extraordinário precipitado insolúvel com $\mathrm{Ca}^{2+}$. Esse produto tem a capacidade de inibir a atividade enzimática dos fungos e é um composto solúvel em água, capaz de formar complexos com o cálcio, responsável pela formação de cristais de oxalato de cálcio.

Green III, Lebow e Yoshimura (2000) também testaram a eficiência do NHA contra cupins, em blocos de madeira de Pinus sp. expostos à degradação por esses agentes. Os autores observaram que a concentração de $0,5 \%$ demonstrou os melhores resultados, tanto em perda de peso como também na taxa de mortalidade dos cupins, sendo que as concentrações podem variar entre 0,5 e $1,0 \%$. Os autores constataram ainda que essas concentrações ofereceram proteção completa contra Reticulitermes flavipes, porém, para Coptotermes formosanus houve perdas de 6,0 e 6,2\% de peso da madeira, respectivamente.

Crawford e Green III (1999) realizaram testes de campo, comparando o comportamento do NHA e do CCA, e constataram que a toxicidade do NHA é 130 vezes menor que a do cobre para testes de $\mathrm{LC}_{50}$. Com relação às perdas de cobre, no NHA estas são 36 vezes maiores que as perdas do cobre do CCA, em meio aquático. Porém, apesar da lixiviação ser maior no NHA, o fato da toxicidade deste ser 52 vezes menor que a do segundo, torna este com poucas restrições para o uso aquático. De maneira geral, o uso de NHA, em estacas de pinus (yellow pine), em concentrações de 0,5 a $1 \%$, apresentou a mesma resistência à degradação que as tratadas com CCA. Nesse mesmo experimento, os autores constataram ainda que o produto não inibiu o desenvolvimento de fungos manchadores, emboloradores, fungos ascomicetos e cupins da espécie Coptotermes formosanus, o que suporta a hipótese deste produto não ser de amplo espectro. Nesse aspecto, Green III et al. (1997) concordam com os autores anteriores, afirmando que o NHA não é efetivo contra todos os tipos de fungos, sendo apenas eficiente contra fungos de podridão parda e branca, que dependem do mecanismo via ligação do cálcio e interface com o ciclo do cálcio.

\section{Outros produtos potenciais}

\footnotetext{
${ }^{5}$ Hyde, S.M., Wood, P.M. A model fro attack at a distance from the hyphae based on studies with the brown rot Coniophora puteana. The International Research Group on Wood Preservation. 1995. Document n ${ }^{\circ}$ IRG/WP 95-10104.

${ }^{6}$ Backa, S., Grirer, J., Reitberger, Y., Nilsson, T. Hydroxyl radical activity in brown-rot fungi by a new chemiluminescence method. Holzforschung, 46, p- 61-67. 1992.

${ }^{7}$ Espejo, E., Agosin, E. Production and degradation of oxalic acid by Brown-rot fungi. Applied and Environmental microbiology, 57, p. 1980-1986. 1991.

${ }^{8}$ Green, F., Larsen, M.J., Winandy, J.F., Highley, T. L. Role of oxalic acid in incipient brown-rot decay. Material und Organismen, 26. p. 191-213. 1991.

${ }^{9}$ Schmidt, C.J., Whitten, B.K., Nicholas, D.D. A proposed role for oxalic acid in non-enzimatic wood decay by brown-rot fungi. Proceedings od American Wood Preservation Association, 77. p. 157-164. 1981.

${ }^{10}$ Beck, S. Oxydimetrische titrationen in Alkalischen losungen mit kaliumcupri-3-perjodat (Percuprimetric). Microchemica Acta, 36/37. p-245-250. 1951.
} 
Entre os compostos alternativos para o tratamento da madeira que tiveram resultados interessantes está o Ibuprofeno (CLAUSEN, 1996). O autor menciona que esse composto tem sido utilizado como preservante de cavacos, na indústria de celulose e para a conservação de grãos. Além das propriedades antiinflamatórias e analgésicas, o produto tem provado ter propriedade fungicida contra podridão parda, mas não contra podridão branca.

Outra alternativa de proteção integral da madeira foi apresentada por Ibach et al. (2000), baseada em tratamentos de acetilação da madeira, iniciada por Rowell e sua equipe nos anos 1987 e 1994, e outro tratamento que consiste na polimerização in situ do composto acrilato de tribiltin (TBTA) nos lumes das fibras. O objetivo principal do tratamento de acetilação é modificar quimicamente os polímeros da parede celular, tirando dos grupos glucosídicos a água necessária para a atividade enzimática dos fungos, inibindo o seu desenvolvimento. O tratamento de polimerização in situ tem por objetivo, além de dar proteção interna às paredes dos lumes, aproveitar a ação tóxica do composto e sua insolubilidade em água para incrementar seu tempo de residência, diminuindo o impacto ambiental provocado pela lixiviação.

As madeiras tratadas no estudo realizado por Ibach et al. (2000) foram submetidas ao ataque de fungos de podridão parda e branca, cupins de solo e de madeira seca, no laboratório e no campo, por um ano. Os dois tipos de tratamento foram efetivos para o controle, em testes realizados no laboratório. No controle de cupins, o tratamento de acetilação não foi efetivo, já que os cupins podem viver em presença de ácido acético, que eles geram em suas colônias.

O tratamento com TBTA teve resultados ótimos, já que provocou $100 \%$ de mortes dos espécimes de cupins nos ensaios de laboratório e somente uma perda de 3 a $6 \%$ de peso, depois de um ano de exposição no campo, em condições extremas.

A partir desse trabalho, Ibach e Rowell uniram esforços para desenvolver algo que definiram como "Polímeros bioativos". O polímero bioativo pode se formar por condensação de grupos bioativos na molécula do polímero ou por sínteses do monômero bioativo, para então ser polimerizado in situ, nos lumes das células na madeira. Os autores publicaram, no ano 2001, dois trabalhos seriados sobre esse item. No primeiro, sintetizaram seis polímeros bioativos: acrilato de pentaclorofenol (PCPA); acrilato de 8-hidroxiquinolina (HQA); dietil N,N-bis (acriloxietil) aminometil fosfatado (Fyrol seis acrilato) F6A; acrilato de 5,7-dibromo-8-hidroxiuinoline (DBHQA) e acrilato de tetraboromobisfenol (TBBPAA). Os primeiros cinco polímeros bioativos mostraram bons rendimentos de incorporação in situ na madeira, com exceção do TBBPAA. No segundo trabalho da série, a madeira de alburno de Pinus foi tratada com diferentes concentrações dos seis polímeros (1-20\%) e um agente iniciador da polimerização (1-50\%), mais $0,1 \%$ de um agente catalisador da polimerização. Blocos de madeira foram tratados com os diferentes acrilatos, sem agente iniciador da polimerização e com diferentes concentrações dele. Mais uma vez, foi determinada a retenção após o tratamento, observando que, para a maioria dos agentes bioativos que não foram polimerizados, a perda de peso foi maior. Nas provas de resistência ao ataque de fungos, o teste foi realizado apenas para o fungo de podridão parda (Gloeophylum tradeum), por um período de 12 semanas, e só os polímeros bioativos TBTA, HQA e DBHQA mostraram resistência.

A modificação da madeira, química ou fisicamente, alterando sua estrutura para torná-la resistente à biodegradação sem o uso dos biocidas, seria o ideal. Vários processos químicos foram desenvolvidos, mas nenhum provou ter a relação custo-benefício favorável. Os tratamentos físicos que usam o calor na ausência do oxigênio mostraram-se promissores, mas não demonstraram desempenho suficiente, além de reduzirem a resistência da madeira. Esses processos são relativamente complexos e caros, e os detalhes ainda são mantidos em segredo pelas companhias envolvidas. Se esses processos se tornarem viáveis economicamente, poderão eliminar os problemas ambientais e de saúde relacionados às outras formas de tratamento (Strategis, 2004).

\section{Dificuldades para a colocação de novos produtos no mercado}

Para o desenvolvimento de novos produtos, para o tratamento preservativo da madeira, dois fatores são cruciais: as viabilidades técnica e econômica para sua utilização. Dentro desse contexto, é evidente que já estão incluídos e devem ser considerados os impactos ambientais e à saúde das pessoas e animais.

Segundo Forest and Wildlife (2004b), os esforços para desenvolver novos preservantes para madeira, ambientalmente corretos, são seriamente dificultados pelo período de tempo prolongado requerido para realizar a avaliação e estabelecimento da eficiência dos produtos potenciais. Atualmente, a avaliação da deterioração da madeira em laboratório e nos testes de campo é feita geralmente determinando-se a perda de massa e pela inspeção visual, respectivamente. Porém, esses métodos não são 
eficientes para a avaliação dos estágios avançados da deterioração, porque não detectam as reações químicas não-enzimáticas que os fungos utilizam para abrir a estrutura da madeira, antes do início das reações enzimáticas extracelulares subseqüentes. Dessa forma, os testes realizados em laboratório e em campo não refletem de forma real a eficiência dos produtos, pois são pouco representativos das condições de trabalho que a madeira tratada irá enfrentar.

Em estudos recentes, realizados pelo Laboratório de Produtos Florestais dos Estados Unidos, demonstrou-se que os estágios avançados da deterioração têm um efeito drástico nas propriedades mecânicas da madeira. Portanto, testes dessas propriedades podem fornecer uma medida excelente da extensão do ataque na madeira. Essa pesquisa mostrou que os testes de resistência à flexão estática, compressão e tração fornecem boas medidas da deterioração da madeira (FOREST AND WILDLIFE, 2004b).

A resistência à flexão estática tem boa relação com a degradação por podridão mole. Já a resistência à compressão transversal tem boa relação com a degradação por basidiomicetos. Além disso, os estudos mostraram que a resistência à tração pode também ser um bom indicador do ataque fúngico na madeira. Essa pesquisa tem o potencial de reduzir significativamente a quantidade de tempo requerida para avaliar os novos preservantes de madeira, acelerando significativamente seu desenvolvimento. Um bom exemplo do uso dos testes das propriedades mecânicas é o da perda da resistência à compressão, para medir a quantidade de deterioração nos testes de laboratório. Usando esse método novo, é possível reduzir o tempo do teste de aproximadamente 14 para 6 semanas (FOREST AND WILDLIFE, 2004b).

Nesse sentido, prevê-se que, quando esses novos métodos estiverem totalmente desenvolvidos, ocorram reduções similares de tempo em outros testes de laboratório e de campo, assegurando com maior rapidez a eficiência dos novos produtos preservativos de madeira (FOREST AND WILDLIFE, 2004b).

\section{Considerações finais}

Com relação aos produtos tradicionais, o CCA, pentaclorofenol e creosoto continuam sendo os produtos mais utilizados. Apesar de serem produtos com maior toxidade para outros organismos e de apresentarem problemas para a eliminação da madeira tratada, são os que tem preços mais competitivos. Além disso, segundo Pitcher (1992), os métodos tradicionais de aplicação de produtos preservantes são as barreiras mais comuns para a adaptação de novas tecnologias ou do uso de novos biocidas pelos usuários, além dos custos de desenvolvimento, testes e as exigências de regulamentação para um simples composto poderem representar um custo de milhões de dólares. Assim, qualquer outro método ou mudança de método são vistos com pessimismo pela indústria. Esses fatores limitam o desenvolvimento de novos produtos. Dessa forma, a educação e treinamento dos trabalhadores envolvidos no tratamento da madeira são necessários para romper as barreiras tradicionais.

Analisando-se as novas alternativas de produtos para o tratamento preservante, os estudos já realizados para a utilização de substâncias extraídas das próprias plantas demonstraram que o uso conjunto de substâncias antioxidantes das plantas, quelantes (derivados dos extrativos fenólicos) e biocidas orgânicos comerciais proporcionam maior proteção à madeira do que o uso isolado do biocida orgânico. Esse método poderia contribuir tanto para a redução do custo de tratamento, uma vez que existem no mercado antioxidantes de baixo custo, já aprovados, como para a redução dos impactos ambientais oriundos do tratamento da madeira.

As pesquisa relativas ao uso de extrativos das plantas desenvolvidas atualmente indicam que os estilbenóides (derivados da pinosilvina), os compostos fenólicos, fenil, éteres e aldeídos são efetivos no controle do ataque de podridão parda, sendo que os três últimos também são efetivos para o controle de fungos manchadores. Os estilbenóides não são eficientes contra a podridão branca, e os demais não atuam sobre cupins. Em contrapartida, a resina de Parthenium argentatum apresenta maior eficiência contra cupins e contribui para a diminuição do ataque de fungos apodrecedores.

A utilização de microorganismos no controle da degradação da madeira tem se mostrado promissora, principalmente através da utilização de metabólitos produzidos por fungos e bactérias. Esses agentes se mostraram efetivos na inibição do ataque dos fungos emboloradores, manchadores e apodrecedores da madeira.

De maneira geral, em se tratando de estratégias biológicas de controle, Pitcher (1992) menciona que os perigos para os operadores desses produtos são muito menores do que o uso dos produtos químicos. As estimativas dos custos de fabricação, testes de laboratório e avaliações de campo podem ser elevadas. Os custos de operação (pulverização e submersão) são comparáveis com os dos produtos químicos atuais, e os perigos ambientais são baixos. 
Os produtos que inibem a ação enzimática dos fungos e outros agentes degradadores, como cupins têm um histórico recente com relação às pesquisas realizadas, sendo que muitos mecanismos envolvidos no processo ainda precisam ser determinados. Além disso, o principal produto testado até o momento, o NHA, não tem amplo espectro de ação, restringindo-se aos agentes que dependem do ciclo do cálcio para agir na degradação da madeira.

Além das possibilidades já mencionadas, outros produtos potenciais estão sendo desenvolvidos. Porém nesses casos as pesquisas ainda são muito recentes, e as informações tanto técnicas quanto econômicas são difíceis de ser conseguidas.

Assim, pode-se concluir que o direcionamento das pesquisas tem objetivado sempre a redução dos impactos ambientais e os cuidados com a saúde das pessoas e outros animais. Além disso, um ponto que precisa ser desenvolvido são as metodologias de teste dos novos produtos para a redução do tempo e dos custos para o seu registro, sem que seja comprometida a qualidade dos novos produtos preservantes da madeira.

\section{REFERÊNCIAS}

BACKA, S.; GRIRER, J.; REITBERGER, Y.; NILSSON, T. hydroxyl radical activity in brown-rot fungi by new chemiluminescence method. Holzforschung, v. 46, p 61-67, 1992.

BULTMAN, J. D.; GILBERTSON, R. L; ADASKAVEG, J.; AMBURGEY, T. L.; PARIKH, S. V.; BAILEY, C. A. The efficacy of guayule resin as a pesticide. Bioresource Technology, v. 35, p. 197-201. 1991.

CELIMENE CATHERINE, JESSI A. MICALES, LESLIE FERGE, RAMOND A. YOUNG. Efficacy of pinosylvins against white-rot and brown-rot fungi. Holzfurschung, v. 53, n. 5, p. 491-497, 1999.

CLAUSEN, C. A. Ibuprofen inhibits in vitro growth of brown-rot fungi. The International Research group on wood preservation. Section 1. 1996. Document $n^{\circ}$ IRG/WP/96-10160.

CRAWFORD, D. M.; GREEN III, F. Protection of southern pine using N,N- Naphthaloylhydroxylamine: Field tests, soft-rot cellars and aquatic bioassay leach testing. In: $30^{\text {th }}$ Annual Meeting The international research group on wood preservation. Section 3 - Wood Protecting Chemicals. 1999, Rosenheim, Germany. Proceedings...,. Rosenheim, Germany, 1999. p.1-8.

CROAN, S. Environmentally benign biological wood preservatives by Streptomyces rimosus, SC-36. In: $28^{\circ}$ Annual Meeting Whistler. 1997, Canadá. Anais.... Canadá. 1997.

FOREST AND WILDLIFE. Making Wood More Durable Research Center. Department of Products Research. Mississipi State University. Disponível em: <http://www.cfr.msstate.edu/fwrc/forestp/ urable.htm >. Acesso em: 02 nov. 2004 (a).

FOREST AND WILDLIFE. New Methods for Accelerating the Development of Wood Preservatives. Research Center. Department of Products Research. Mississipi State University. Disponível em: $<$ http://www.cfr.msstate.edu/fwrc/forestp/durable.htm>. Acesso em: 02 nov. 2004 (b).

FREEDONIA GROUP. World Biocides to 2006 - Market Size, Market Share, Demand Forecast and Sales. Disponível em: <http://www.freedoniagroup.com/World-Biocides.html.>. Acesso em: 02 nov. 2004.

GREEN III, F.; KUSTER, T. A.; FERGE, L.; HIGHLEY, T. L. Protection of Southern Pine from fungal decay and termite Damage with N,N-Naphthaloylhydroxylamine. International Biodeterioration e Biodegradation. v. 39, n. 2/3, p 103-111, 1997.

GREEN III, F.; LEBOW; S.; YOSHIMURA, T. Inhibition of termite damage by N'Nnapthaloylhydroxilamine (NHA): Reticulotermes flavipes (Kollar) vs. coptotermes formasanus Shiraki. The interntional research group on wood preservation. 2000. IRG/WP 00-10354.

HIGHLEY, T. control of wood decay by Trichoderma (Gliocladium) virens. I. Antagonistic properties. In: International Research (IRG) meeting, 1995, Helsinger, Denmark. Anais... Helsinger, Denmark, 1995. p. 79-89. 
HIGHLEY, T. L.; PADMANABHA, H. S. A.; HOWELL, C. R. Antagonistic properties of Gliocladium virens against wood attacking fungi. In: 27 th Annual Meeting. The interntional research group on wood preservation, 1996, Guadaloupe. Anais...Guadaloupe, 1996.

IBACH, R. E.; ROWELL, R. M. Wood preservation based on in situ polymerization of bioactive monomers. Part 1. Synthesis of Bioactive monomers, wood treatments and microscopic analysis. Holzforschung. v. 55, n. 4, p-358-364. 2001.

IBACH, R. E.; ROWELL, R. M. Wood preservation based on in situ polymerization of bioactive monomers. Part 2. Fungal resistence and thermal properties of treated wood. Holzforschung. v. 55, n 4, p $-365-372.2001$

IBACH, R. E.; HADI, Y. S.; NANDIKA, D.; YUSUF, S.; INDRAYANI, Y. Termite and fungal resistance of in situ polymerized tributyltin acrylate and acetylate Indonesian and USA wood. Section 3. International Research group on wood preservation. 2000. Document $n^{\circ}$ IRG/WP/00-30219.

KARTAL, S. N.; IMAMURA, Y.; TSUCHIYA, F.; OHSATO. K. Preliminary evaluation of fungicidal and termiticidal activities of filtrates from biomass slurry fuel production. Bioresource Technology (Article in press), 2004.

NAKAYAMA, F. S.; VINYARD, S. H.; CHOW, P.; BAJWA, D. S.; YOUNGQUIST, J. A.; MUEHL, J. H.; KRZYSIK, A. M. Guayule as a wood preservative. Industrial Crops and Products. v. 14, p. 105111. 2001

ONUORAH, E. O. The wood preservative potenctials of heartwood extracts of Milicia excelsa and Erythrophleum suaveolens. Bioresource Technology. v.75, p. 171-173. 2000.

PITCHER, J. A. Market Potential for Technological Innovations in Eastern Hardwoods. In: MURDOCH, C.W. Transferring Technologies for Industry, no. 4: Alternatives to Petroleum-Based Biocides for Protecting Hardwood Lumber and Manufactured Products. Hardwood Research Council. University of Maine. Beltsville, 1992.

SCHMIDT, C. J.; WHITTEN, B. K.; ENICHOLAS, D. D. A proposed role for oxalic acid in nonenzimatic wood decay by brown-rot fungi. American Wood Preservation Association, v. 77, p - 157 164. 1981

SCHULTZ, T. P.; NICHOLAS, D. D. Development of environmentally-benign wood preservatives based on the combination of organic biocides with antioxidants and metal chelators. Phytochemistry, v. 61, p 555-560. 2002.

STRATEGIS. Preservative Technology. Disponível em: $<$ http://strategis.ic.gs.ca/epic/internet/infiif.nsf/en/fb01463e. html>. Acesso em: 02 nov. 2004.

SUTTIE, E. D.; ORLER, R. J.; EWOOD, P. M. Preliminary studies of the performances of iron chelatiors as inhibitors of brown-rot (Cniophora puteana) attack. International Research group on wood preservation. 1996. Document $\mathbf{n}^{\mathbf{0}}$ IRG/WP/96-10185.

VODA KARMEN, BOJANA BOH, MARGARETA VRTACNIK, FRANC POHLEVEN. Effect of the antifungal activity of oxygenated aromatic essential oil compounds on the white-rot Trametes versicolor and the brown-rot Coniphora puteana. International Biodeterioration and biodegradation, v. 51, p. 51-59.2003.

WORLD BIOCIDES. Disponível em: < http://www.the-infoshop.com/study/fd12429_biocides.htm>. Acesso em: 02 nov. 2004. 